\title{
Synthetic regulatory RNAs selectively suppress the progression of bladder cancer
}

\author{
Chengle Zhuang ${ }^{1} \mathbb{D}$, Xinbo Huang ${ }^{1}$, Changshui Zhuang ${ }^{1}$, Xiaomin Luo ${ }^{1}$, Xiaowei Zhang ${ }^{2}$, Zhiming Cai ${ }^{1}$ \\ and Yaoting Gui ${ }^{1 *}$
}

\begin{abstract}
The traditional treatment for cancer is lack of specificity and efficacy. Modular synthetic regulatory RNAs, such as inhibitive RNA (iRNA) and active RNA (aRNA), may overcome these limitations. Here, we synthesize a new iRNA to bind the upstream activating sequence (UAS) of a minimal promoter that drives expression of artificial miRNAs (amiRNAs) targeting MYC, which represses the binding interaction between UAS and GAL4 fusion protein (GAL4-VP64) in GAL4/UAS system. The aRNA driven by a tumor-specific mutant human telomerase reverse transcriptase ( $h T E R T$ ) promoter is created to interact with iRNA to expose UAS again in bladder cancer. Without the aRNA, mRNA and protein levels of MYC, cell growth, cell apoptosis and cell migration were no significance in two bladder cancer cell lines, T24 and 5637, and human foreskin fibroblast (HFF) cells. The aRNA significantly inhibited the expression of MYC in mRNA and protein levels, as well as the proliferation and migration of the cancer cells, but not in HFF cells. These results indicated that regulatory RNAs selectively controlled the expression of amiRNAs and ultimately suppress the progression of bladder cancer cells without affecting normal cells. Synthetic regulatory RNAs might be a selective therapeutic approach for bladder cancer.
\end{abstract}

Keywords: Bladder cancer, Human telomerase reverse transcriptase, Artificial miRNA, GAL4

\section{Introduction}

Bladder cancer is one of the most common tumors all over the world [1]. Surgery, radiation therapy and chemotherapy are major therapies for the treatment of bladder cancer while they have inevitable side effects because of lack of specificity [2,3]. Thus, we should focus on an effective and tumor-specific therapeutic method for bladder cancer.

MicroRNA (miRNA) is a type of endogenous noncoding RNAs and also a key mediator of RNA interference (RNAi) in eukaryotes [4-6]. Many protein-coding genes are influenced by miRNAs through target-sequence interaction [4]. Complete or partial pairing of the miRNA complex to the target mRNA transcript causes RNA degradation or translational inhibition [7]. Studies show that based on the pri-miR-155 backbone, artificial miRNAs (amiRNAs) were created through inserting a series of miRNA precursors with different stem sequences, which

\footnotetext{
* Correspondence: guiyaoting2007@aliyun.com

${ }^{1}$ Guangdong and Shenzhen Key Laboratory of Male Reproductive Medicine and Genetics, Institute of Urology, Peking University Shenzhen Hospital, Shenzhen-Peking University-the Hong Kong University of Science and Technology Medical Center, Shenzhen 518000, People's Republic of China Full list of author information is available at the end of the article
}

generated efficient mature miRNA [8, 9]. In addition, a subset of non-coding RNAs, regulatory RNAs, repress or activate translation through sensing environmental signals or other RNA molecules [10].

GAL4, a yeast transcriptional activator, and upstream activating sequence (UAS) that is the specific recognition sequence of GAL4 or GAL4-VP64 fusion protein, are two components in GAL4/UAS system [11]. This system operates not only in yeast but also in various mammal cells $[12,13]$. The UAS, a minimal promoter, cannot drive the expression of downstream targeting genes until the binding of GAL4 protein or GAL4-VP64 fusion protein [13]. Moreover, to develop a kind of cancer-specific treatment, we try to use tumor-specific elements to achieve this purpose. The human telomerase reverse transcriptase (hTERT), one of the subunits of telomerase, exists in most tumors but not normal tissues $[14,15]$. Previous studies show that compared with the wild-type $h T E R T$ promoter, the mutant $h T E R T$ promoter could enhance the expression of $h T E R T$ or downstream genes and still maintain its tumor-specific feature [16-18]. We pick the tumor-specific element, mutant $h T E R T$ promoter, from a previous study [19]. In their study, they utilized the mutant $h T E R T$ to drive expression of BCL2 shRNA. However, 
their driven efficiency was still not very high and stable expression of BCL2 shRNA may do harm to cells. Compared with their work, we may overcome these limitations.

Using the above elements, we could construct artificial devices with novel functions according to the principles of synthetic biology [17, 20, 21]. Synthetic devices have been used to regulate gene expression or control the biological phenotypes of cancer cells [22, 23]. Synthetic amiRNA, one of the synthetic devices, could knockdown expression of genes with several advantages, including co-expression with a gene of interest, stable expression and low toxicity [24-28]. Wang, et al. synthesized amiRNA clusters and used them as powerful tools for multiplex gene knockdown at the posttranscriptional level [9]. In this study, we construct and synthesize regulatory RNAs to control the gene expression. The inhibitive RNA (iRNA) binds UAS so GAL4-VP64 cannot recognize UAS. The active RNA (aRNA) which is constructed according to the previous study [10] interacts with iRNA tightly and UAS is exposed again, and finally GAL4-VP64 binds UAS to activate amiRNAs targeting MYC. MYC was one of the most well-known deregulated oncogenes and the third most amplified gene in human cancer $[29,30]$. In bladder cancer, increase of $M Y C$ copy number occurred before muscle invasion and correlated with grade [31]. Furthermore, MYC was regarded as an independent predictor of progression-free and cancer-specific survival [32]. Thus, we choose MYC as the therapeutic target in this study.

In our study, we constructed synthetic artificial miRNA devices driven by UAS to suppress the expression of the $M Y C$ oncogene in bladder cancer. As mentioned above, synthetic iRNA block UAS from binding the GAL4-VP64 fusion protein. And results of in vitro and in vivo experiments showed that the GAL4-VP64 fusion protein interacts with UAS again when aRNA expressed. In short, synthetic regulatory RNAs selectively inhibit the progression of bladder cancer through controlling the expression of amiRNAs targeting MYC.

\section{Materials and methods}

Cell lines and cell culture

Human bladder cancer cell lines (T24 and 5637) and human foreskin fibroblast (HFF) cells were purchased

Table 1 Relative sequences in this study

\begin{tabular}{|c|c|}
\hline Name & Relative sequence $\left(5^{\prime}-3^{\prime}\right)$ \\
\hline NC amiRNA & $\begin{array}{l}\text { CTCGAGAAGGTATATTGCTGTTGACAGTGAGCGCATTCTCCGAACGTGTCACGTATAGTG } \\
\text { AAGCCACAGATGTATACGTGACACGTTCGGAGAATTGCCTACTGCCTCGCTTCAAGGTA } \\
\text { TATTGCTGTTGACAGTGAGCGCATTCTCCGAACGTGTCACGTATAGTGAAGCCACAGATG } \\
\text { TATACGTGACACGTTCGGAGATTGCCTACTGCCTCGGCGGCCGC }\end{array}$ \\
\hline MYC amiRNA & $\begin{array}{l}\text { CTCGAGAAGGTATATTGCTGTTGACAGTGAGCGCACAGAAATGTCCTGAGCAATATAGTG } \\
\text { AAGCCACAGATGTATATTGCTCAGGACATTCTGTIGCCTACTGCCTCGCTCCTCCTTC } \\
\text { AAGGTATATTGCTGTTGACAGTGAGCGCATGGACAGTGTCAGAGTCTATAGTGAAGCCAC } \\
\text { AGATGTATAGACTCTGACACTGTCCATTGCCTACTGCCTCGGCGGCCGC }\end{array}$ \\
\hline iRNA & GAAUUCCGGAGGUCAGAACUCUUGG \\
\hline aRNA & $\begin{array}{l}\text { CGCCAAGAGUUCUGUCCUCCGGUGGUGGUUAAUGAAAAUUAACUUACUAUACCAUAUA } \\
\text { UCUCUAGA }\end{array}$ \\
\hline $\begin{array}{l}\text { mutant } \\
\text { hTERT promoter }\end{array}$ & $\begin{array}{l}\text { GGCCCCTCCCTCGGGTIACCCCACAGCCTAGGCCGATTCGACCTCTCTCCGCTGGGGCC } \\
\text { CTCGCTGGCGTCCCTGCACCCTGGGAGCGCGAGCGGCGCGCGGGCGGGGAAGCGCGG } \\
\text { CCCAGACCCCCGGGTCCGCCCGGAGCAGCTGCGCTGTCGGGGCCAGGCCGGGCTCCCA } \\
\text { GTGGATTCGCGGGCACAGACGCCCAGGACCGCGCTCCCCACGTGGCGGAGGGACTGGG } \\
\text { GACCCGGGCACCCGTCCTGCCCCTCACCTTCCGGCTCCGCCTCCTCCGCGCGGACCCCG } \\
\text { CCCCGTCCCGACCCCTTCCGGGTTCCGGCCCAGCCCCTCCGGGCCCTCCCAGCCCCTC } \\
\text { CCCTCCCTICCGGGGCCCCGCCCTCTCCTCGCGGCGCGAGTITCCGGCAGCGCTGCGTC } \\
\text { CTGCTGCGCACGTGGGAAGCCCTGGCCC CGGCCACCCCCGCG }\end{array}$ \\
\hline UAS & CGGAGTACTGTCCTCCG \\
\hline GAL4-VP64 & $\begin{array}{l}\text { ATGAAGCTACTGTCTTCTATCGAACAAGCATGCGATATTTGCCGACTTAAAAAGCTCAAG } \\
\text { TGCT CCAAAGAAAAACCGAAGTGCGCCAAGTGTCTGAAGAACAACTGGGAGTGTCGCT } \\
\text { ACTCTCCCAAAACCAAAAGGTCTCCGCTGACTAGGGCACATCTGACAGAAGTGGAATCA } \\
\text { AGGCTAGAAAGACTGGAACAGCTATTCTACTGATIITCCTCGAGAAGACCTGACATG } \\
\text { ATTITGAAAATGGTTCTITACAGGATATAAAAGCATTGTTAACAGGATTATTGTACAAGA } \\
\text { TAATGTGAATAAAGATGCCGTCACAGATAGATTGGCTTCAGTGGAGACTGATATGCCTCTA } \\
\text { ACATTGAGACAGCATAGAATAAGTGCACATCATCATCGGAAGAGAGTAGTAACAAAGGT } \\
\text { CAAAGACAGTTGACTGTATCGGGTCCGGACGGGCTGACGCATGGACGATIITGATCTG } \\
\text { GATATGCTGGGAAGTGACGCCCTCGATGATTTGACCTGACATGCTGGTTCGGATGCCC } \\
\text { TTGATGACTTGACCTCGACATGCTCGGCAGTGACGCCCTTGATGATTCGACCTGGACAT } \\
\text { GCTGATTAAC }\end{array}$ \\
\hline
\end{tabular}


from the Institute of Cell Research, Chinese Academic of Sciences, Shanghai, China. The normal bladder epithelium SV-HUC-1 cell line was established by transformation of human normal ureter tissue with SV40 virus, and purchased from American Type Culture Collection (ATCC). T24 and HFF cells were cultured in DMEM (Invitrogen, Carlsbad, CA, USA) with $10 \%$ fetal bovine serum (FBS). The 5637 cells were maintained in 10\% FBS RPMI-1640 media (Invitrogen, Carlsbad, CA, USA). The SV-HUC-1 cells were grown according to the manufacturer's protocol. The cells were cultured at $37^{\circ}$ $\mathrm{C}$ in a humidified atmosphere of $5 \% \mathrm{CO}_{2}$ in an incubator.

\section{Patient samples}

Thirty-nine pairs of bladder cancer tissues and matched para-carcinoma tissues were resected from patients diagnosed with bladder cancer. Samples were treated with other necessary procedures according to a previous study [33]. This study was admitted by the Institutional Review Board of Peking University Shenzhen Hospital.

\section{Creation of iRNA, aRNA and artificial miRNAs}

To construct a vector that expresses iRNA, the sequence of iRNA was inserted into the pcDNA3-EGFP vector (Addgene \#13031) between the restriction sites XhoI and XbaI. To create vectors expressing aRNA, we used the mutant $h T E R T$ promoter and aRNA to replace CMV promoter and EGFP respectively in the pcDNA3-EGFP vector. GAL4-VP64 displaced EGFP in pcDNA3-EGFP vector to create pcDNA3-GAL4-VP64 vector. Besides, UAS and related artificial microRNAs were designed, synthesized and inserted between the restriction sites BbsI and BstBI into the pcDNA3-GAL4-VP64 vector. In dual luciferase reporter assays, UAS replaced the SV40 promoter in the siCHECK ${ }^{\mathrm{m}}-2$ vector (Promega, Madison,
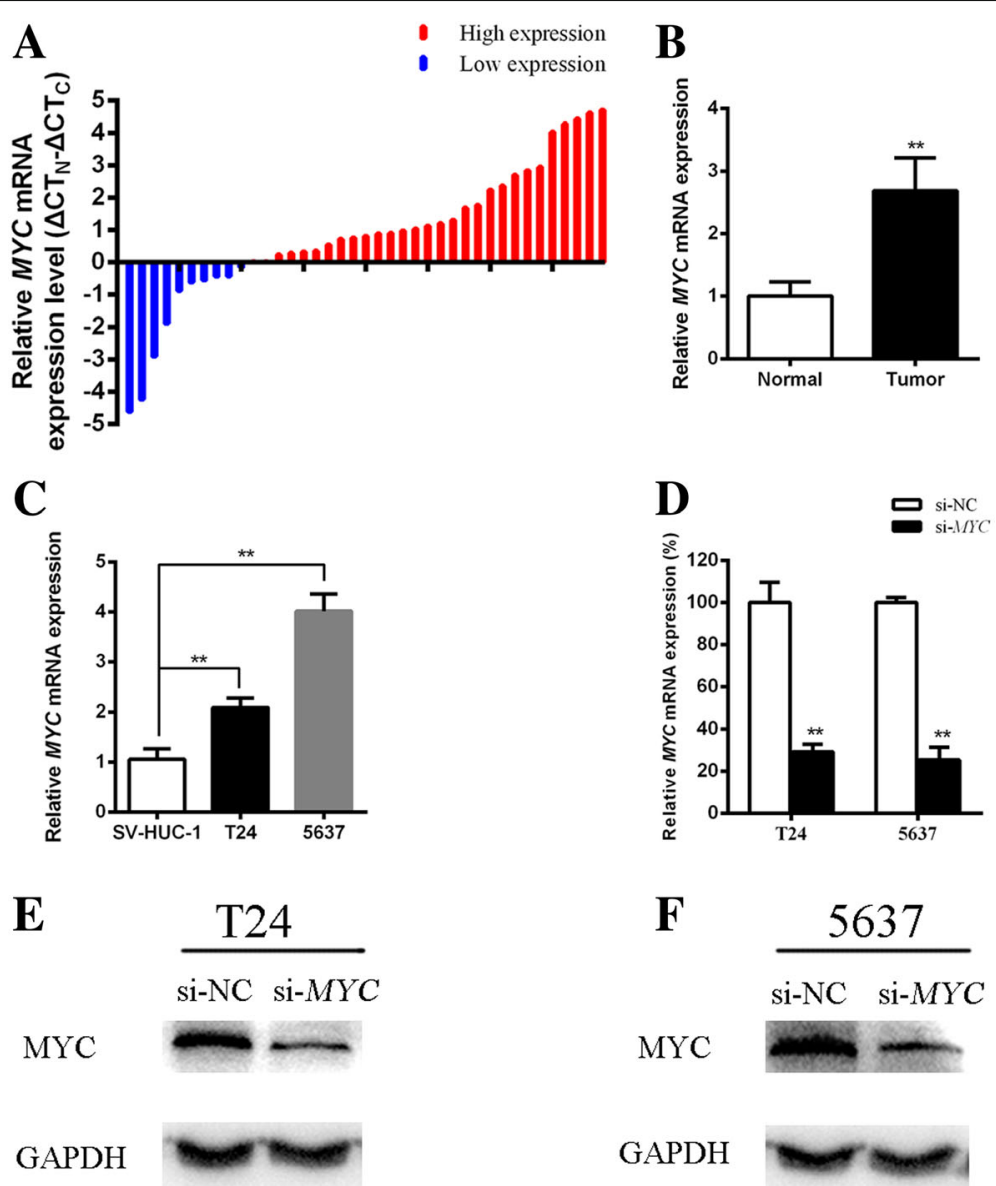

Fig. 1 The expression of MYC was upregulated in bladder cancer tissues and cells, and siRNA was able to knockdown the expression of this gene in cells. (a) qRT-PCR was used to detect the expression of MYC in bladder cancer tissues. $\Delta C T_{N}$ means comparative $C T$ in normal tissue. $\Delta C T_{C}$ represents comparative $C T$ in tumor tissue. (b, c) Compared with the negative control, the expression of MYC mRNA was significantly increased in bladder cancer tissues (b, $P<0.01$ ), bladder cancer cell lines T24 and $5637(C, P<0.01)$. (d) The mRNA expression level of MYC was statistically decreased after transfection of si-MYC in T24 and 5637 cells (both $\mathrm{P}<0.01$ ). (e, f) The protein expression level of MYC was statistically decreased after transfection of si-MYC in T24 (e) and 5637 (f) (both $P<0.01$ ). All data are shown as the mean \pm SD $\left({ }^{*} p<0.05,{ }^{* *} p<0.01\right.$ ); bar, SD 
USA) between the restriction sites BgIII and Nhel. The iRNA can bind UAS while aRNA driven by mutant $h T E R T$ promoter can interact with iRNA. The siRNA duplexes for $M Y C$ and the negative control (indicated as si-MYC and si-NC) were designed and synthesized by GenePharma, Suzhou, China. All of the related sequences were shown in Table 1.

\section{Cell transfection}

The propagated synthetic constructed vectors from E.coli bacteria were extracted using Plasmid Midiprep kits (Promega, Madison, USA). The cells were transfected with specific siRNA or synthetic vectors using Lipofectamine 2000 Transfection Reagent (Invitrogen, Carlsbad, CA, USA) according to the manufacturer's instructions.

\section{Dual luciferase reporter assay}

Cells $\left(1 \times 10^{5}\right.$ per well $)$ were cultured in 24-well plates and transfected with specific siRNA or vectors. After $48 \mathrm{~h}$ transfection, the luciferase activity was measured using the dual luciferase assay system (Promega, Madison, WI, USA) according to the manufacturer's protocol. The experiments were performed at least three times.

\section{RNA extraction and quantitative RT-PCR}

The TRIzol reagent (Invitrogen, Grand Island, NY, USA) was used to extract total RNA from cells after transfection according to the manufacturer's protocol. The cDNA was synthesized from total RNA using PrimeScript RT Reagent Kit with gDNA Eraser (Takara, Dalian, China). The mRNA expression levels of $M Y C$ were measured by quantitative RT-PCR (qRT-PCR) on the Roche lightcycler 480 RealTime PCR System. GAPDH was used as the endogenous control to normalize the data. The primers used were: MYC-forward: 5'-GCAGCTGCTTAGACG CTGGA-3', MYC-reverse: 5'-CGCAGTAGAAATACGGCTGCAC-3'; GAPDH -forward: 5'-CGCTCTCTGCTCCTCCTGTTC3', GAPDH-reverse: 5'-ATCCGTT GACTCCGACCTT CAC-3'. The comparative $\triangle \mathrm{Ct}$ method was used to analyze the relative expression of $M Y C$. All of the experiments were performed at least three times.

\section{Western-blot analysis}

The transfected cells were washed in PBS and lysed in RIPA reagent (Beyotime, Jiansu, China). The bicinchoninic acid quantification assay (Pierce Biotechnolofy, Rockford, IL, USA) was used to calculate the protein concentration. Equal amounts of whole protein extract were electrophoresed on SDS-polyacrylamide gels and transferred to polyvinylidene difluoride membranes using a semi-dry transfer cell (Bio-Rad Laboratories, Hercules, CA, USA). After blocked with $5 \%$ milk, the membranes were incubated over night with specific primary antibodies against MYC (1:1000; Cell Signaling Technology, USA) and GAPDH (1:10,000; Sigma-Aldrich). Horseradish peroxidaseconjugated secondary antibody (Amersham, Piscataway, NJ, USA) was used to incubate the blot for one hour at room temperature on a rocking platform. Finally, signal intensities were quantified using Super Signal chemiluminescence reagents (Pierce).

\section{Cell proliferation assay}

Cell Counting Kit-8 (Beyotime Institute of Biotechnology, Shanghai, China) was used to detect cell proliferation according to the manufacturer's instructions. Cells (4000 per well) were seeded into a 96-well plate. Then, 24,48 or $72 \mathrm{~h}$ after transfection, $10 \mu \mathrm{l}$ of CCK- 8 was added to each well and the cells were incubated for $40 \mathrm{~min}$. Absorbance was detected at a wavelength of $450 \mathrm{~nm}$ using an ELISA microplate reader (Bio-Rad, Hercules, CA, USA). All of the assays were performed in triplicate.

\section{ELISA assay}

Cells were transfected with specific siRNA or vectors. The activity of caspase- 3 represented the levels of apoptosis and was measured using the caspase 3 enzymelinked immunosorbent assay (ELISA) assay kit (Hcusabio, Wuhan, China) according to the manufacturer's protocols. All of the experiments were performed at least three times.

\section{Cell migration assay}

Cells were seeded in 6-well plates to $90 \%$ confluence before transfection. A sterile pipette tip was used to create

Table 2 Correlation between MYC expression and clinicopathological characteristics of bladder cancer patients

\begin{tabular}{|c|c|c|c|c|c|}
\hline \multirow[t]{2}{*}{ Parameters } & \multirow[t]{2}{*}{ Group } & \multirow[t]{2}{*}{ Total } & \multicolumn{2}{|c|}{ MYC expression } & \multirow[t]{2}{*}{$P$ value } \\
\hline & & & High & Low & \\
\hline \multirow[t]{2}{*}{ Age (years) } & $<59$ & 12 & 7 & 5 & \multirow[t]{2}{*}{0.232} \\
\hline & $\geq 59$ & 27 & 22 & 5 & \\
\hline \multirow[t]{2}{*}{ Gender } & Male & 25 & 18 & 7 & \multirow[t]{2}{*}{0.721} \\
\hline & Female & 14 & 11 & 3 & \\
\hline \multirow[t]{2}{*}{ Tumor size $(\mathrm{cm})$} & $<3$ & 21 & 15 & 6 & \multirow[t]{2}{*}{0.726} \\
\hline & $\geq 3$ & 18 & 14 & 4 & \\
\hline \multirow{2}{*}{$\begin{array}{l}\text { Histological grade } \\
\text { (PUNLMP) }\end{array}$} & Low-grade & 19 & 11 & 8 & \multirow[t]{2}{*}{$0.031^{*}$} \\
\hline & High-grade & 20 & 18 & 2 & \\
\hline \multirow[t]{2}{*}{ TNM stage } & $0 / 1$ & 8 & 3 & 5 & \multirow[t]{2}{*}{$0.016^{*}$} \\
\hline & $\|/\| / \| / I V$ & 31 & 26 & 5 & \\
\hline \multirow[t]{2}{*}{ Lymph nodes metastasis } & NO & 33 & 25 & 8 & \multirow[t]{2}{*}{0.636} \\
\hline & N1 or above & 6 & 4 & 2 & \\
\hline
\end{tabular}

PUNLMP papillary urothelial neoplasm of low malignant potential, TNM according to the seventh edition of staging TNM of Union Internationale Contre Le Cancer (UICC) in 2009

${ }^{*} \mathrm{P}<0.05$ was considered significant (Chi-square test between 2 groups) 
a clear line. Twenty-four hours after transfection, the migration distance was measured using the software program HMIAS-2000. The experiments were repeated at least three times.

\section{Xenograft model of tumor growth in vivo}

The experimental procedures were approved by Institutional Ethics Review Board. Male immune-deficient $\mathrm{BALB} / \mathrm{c}$ nude mice (4-5 weeks old) were purchased from Beijing Wei-tong Li-hua Laboratory Animals and
Technology Ltd., Beijing, China. Vectors were packed into lentivirus according to the manufacturer's protocols using Lentiviral Packing Kit, SyngenTech, China. In detail, $10^{7}$ 5637 cells were suspended in $100 \mu$ l Matrigel (BD Biosciences, Franklin Lakes, NJ, USA) and injected subcutaneously into the right armpits of BALB/c nude mice. LV-NC represents NC amiRNA + GAL4-VP64 + iRNA + aRNA. Besides, $M Y C$ amiRNA + GAL4-VP64+ iRNA + aRNA was regarded as the LV-Treatment group. 15 days after implantation, tumor volumes were monitored every 5 days

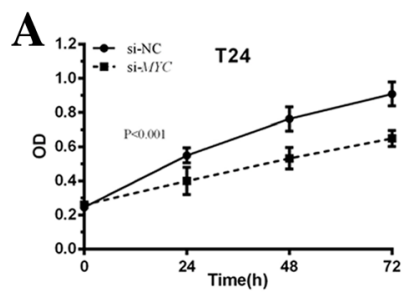

C

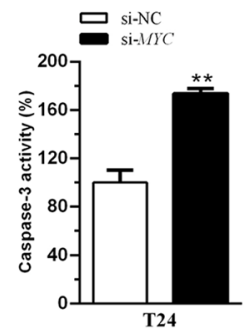

$\mathbf{E}$

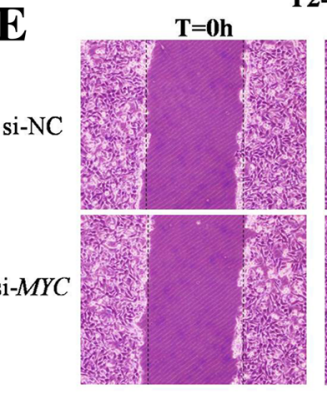

T24
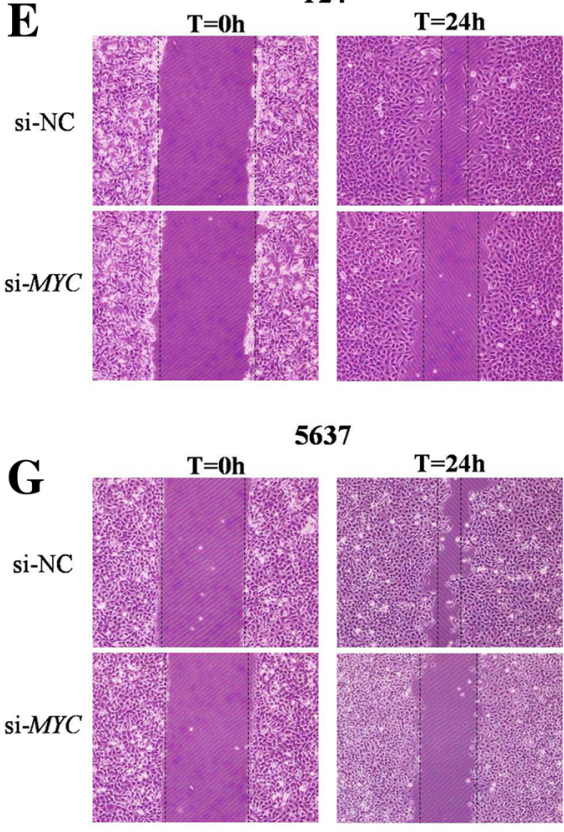

5637

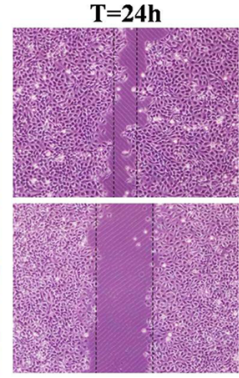

B

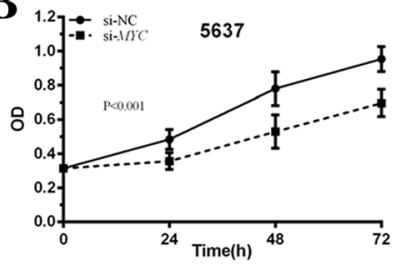

D

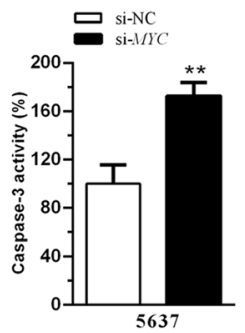

$\mathbf{F}$

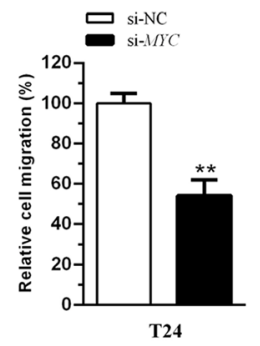

$\mathbf{H}$

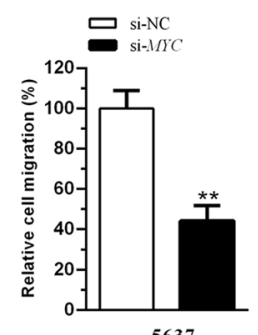

Fig. 2 Effects of MYC on cell proliferation, apoptosis and cell migration in bladder cancer cells. (a, b) Compared with the si-NC group, cell growth was inhibited significantly in the si-MYC group in T24 (A) and 5637 (B) (both $P<0.01$ ). (c, d) Compared with the si-NC group, cell apoptosis was statistically increased in the si-MYC group in T24 (c) and 5637 (D) (both P < 0.01). (e, f) Compared with si-NC, cell migration was significantly suppressed after transfection of si-MYC in T24. (g, h) Compared with the negative control, cell motility was significantly inhibited after transfection of si-MYC in 5637. All data are shown as the mean \pm SD $\left({ }^{*} p<0.05,{ }^{* *} p<0.01\right)$; bar, SD 
over a 2-week period. Tumor volumes were calculated using the formula: $0.5 \times$ length $\times$ width $^{2}$. At the end of the experiment, mice were euthanized, and the subcutaneous weight of each tumor was measured.

\section{Statistical analysis}

All statistical analyses were performed using 21.0 version SPSS computer software (SPSS Inc., Chicago, IL, USA). The data are presented as the mean \pm S.D. and were analyzed using Student's $t$-test or ANOVA. A two-sided value of $P<0.05$ was considered statistically significant.

\section{Results}

\section{MYC is an oncogene in bladder cancer}

Compared with normal counterparts, the MYC mRNA expression level was increased significantly in $74.4 \%$ (29 of 39) of tumor tissues (Fig. 1A). The expression of $M Y C$ in tumors was 2.8 times higher than in paracancer tissues (Fig. 1B, $\mathrm{P}<0.01$ ). Compared with the SV-HUC-1 cell line, MYC expression was upregulated significantly in T24 and 5637 (Fig. 1C, both $P<0.01$ ). As shown in Table 2, high expression of $M Y C$ was significantly correlated with bladder cancer histological grade $(P=0.031)$ and TNM stage $(P=0.016)$. However, there was no association between age, gender, tumor size, lymph node metastasis and $M Y C$ expression level. To investigate the function of this gene in bladder cancer cells, we synthesized specific siRNA duplexes to knockdown $M Y C$ expression. The qRT-PCR results showed that si-MYC, but not si-NC, can dramatically decrease the expression of $M Y C$ in T24 and 5637 (Fig. 1D, both $P<0.01$ ). The Western-blot analysis assay demonstrated that compared with the protein level of MYC in siNC group, the protein level was significantly suppressed in si-MYC group in T24 (Fig. 1E) and 5637 (Fig. 1F). After inhibition of $M Y C$ expression, cell growth was arrested significantly in T24 and 5637 (Fig. 2A and B, both $P<0.001$ ). Additionally, cell apoptosis was significantly promoted after suppression of $M Y C$ expression in T24 and 5637 (Fig. $2 C$ and D, both $P<0.01$ ). In addition, cell migration was repressed after transfection with si-MYC (Fig. 2E, F, G and H, both $\mathrm{P}<0.01$ ). These data suggest that $M Y C$ should be an oncogenic factor in bladder cancer cells.

\section{Schematic strategy for this study and luciferase assay that was used to validate the roles of aRNA and iRNA in bladder cancer cells}

The schematic strategy for the expression of the synthetic artificial miRNA to target oncogenic MYC was

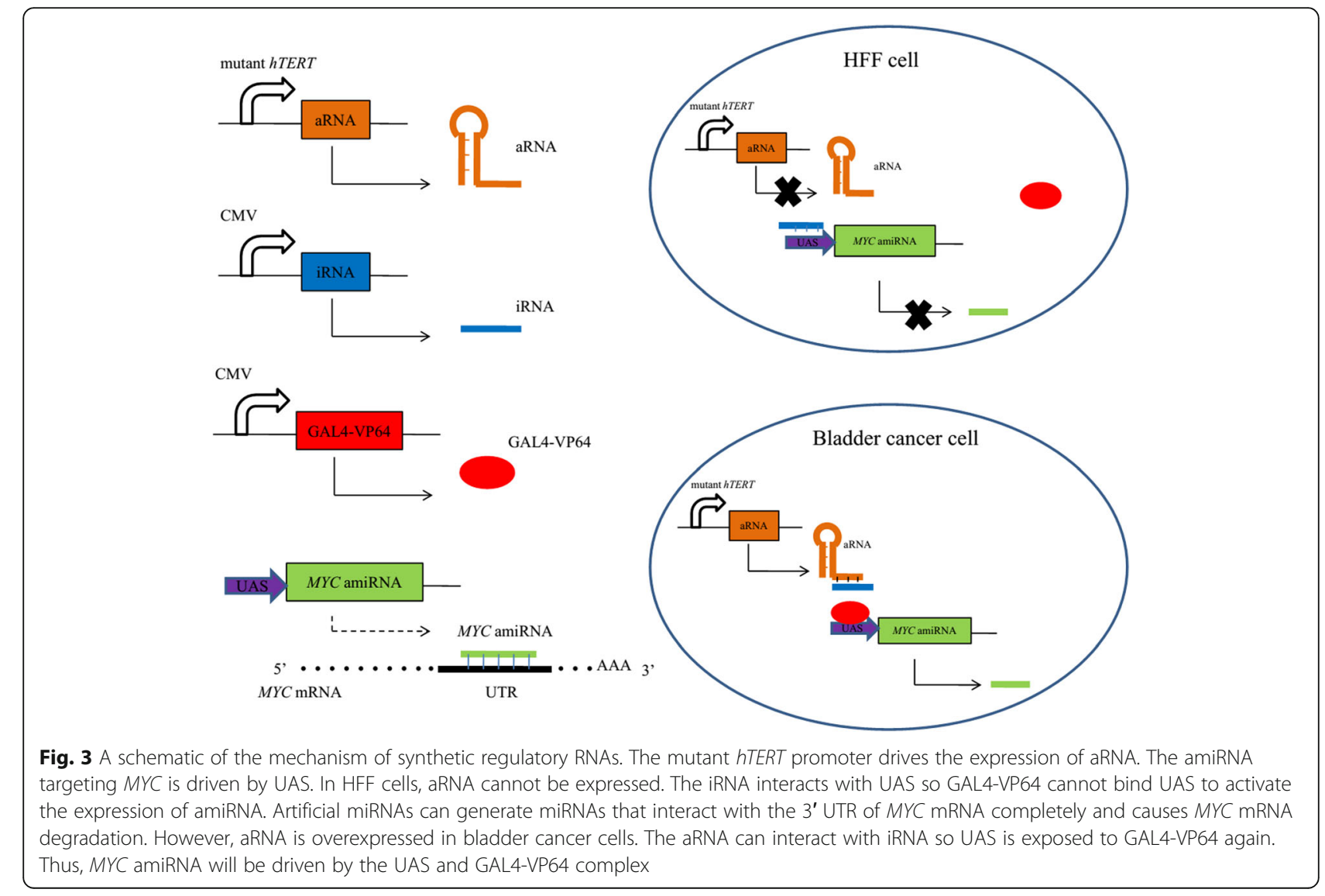


shown in Fig. 3. The amiRNA cannot be expressed without aRNA when the cells were only transfected with GAL4-VP64 and iRNA. The aRNA driven by the tumorspecific mutant $h T E R T$ promoter could be expressed as expected only in bladder cancer cells but not in HFF cells. Artificial miRNAs can generate miRNAs that bind the 3' UTR of MYC mRNA completely and causes MYC mRNA degradation. Thus, we can use this strategy to control the expression of $M Y C$ in bladder cancer cells. To verify whether iRNA can bind aRNA and UAS, we transfected relevant vectors (UAS-luciferase, GAL4-
VP64, iRNA and aRNA) into cells and performed luciferase assays. When aRNA was not expressed in these three cell lines, the luciferase activity was significantly decreased between the NC iRNA group and iRNA group in T24, 5637 and HFF (Fig. 4A, B and C, P < 0.05). The results showed that iRNA can bind UAS so that GAL4VP64 cannot interact with UAS to activate the expression of luciferase. After transfection of aRNA in cells, the luciferase activity did not change in T24 and 5637 between NC iRNA group and iRNA group (Fig. 4B and $\mathrm{C}, \mathrm{P}>0.05)$. However, it was still dramatically decreased
A
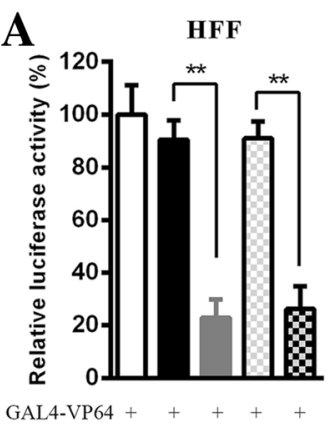

NC iRNA

iRNA

aRNA

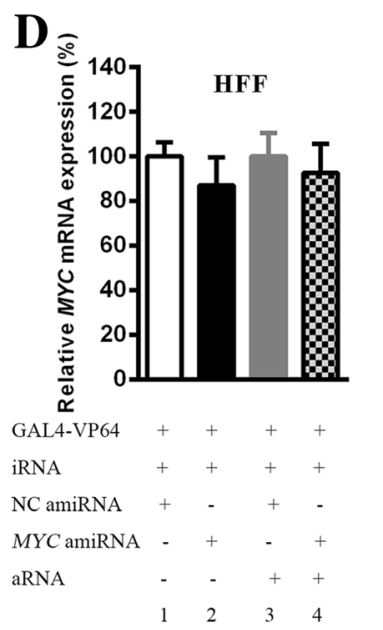

G

HFF

$\begin{array}{llll}1 & 2 & 3 & 4\end{array}$

MYC

GAPDH
B

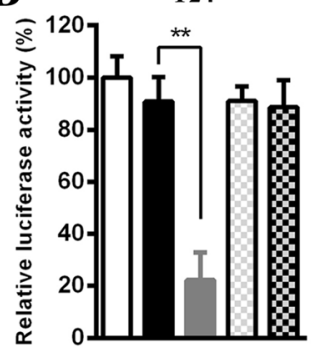

GAL4-VP64 +

NC IRNA

iRNA

aRNA

E

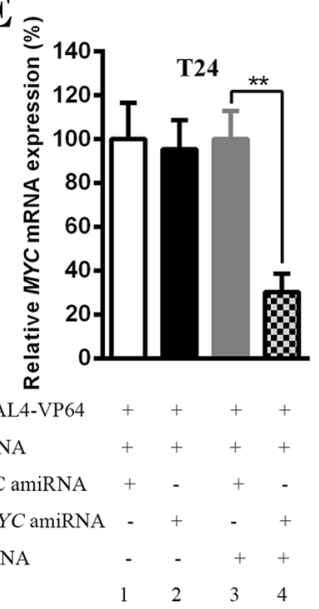

$\mathbf{H}$

T24

$\begin{array}{llll}1 & 2 & 3 & 4\end{array}$

MYC
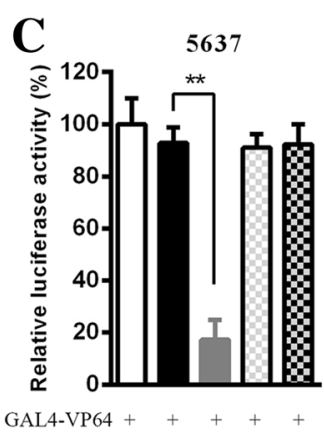

NC iRNA - + - + -

iRNA

aRNA

F

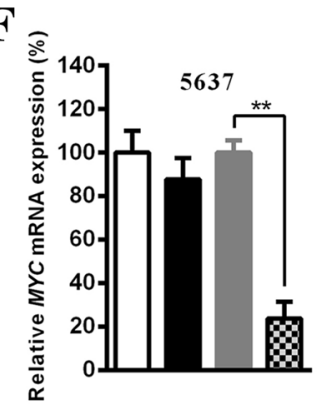

GAL4-VP64

iRNA

NC amiRNA

$M Y C$ amiRNA

aRNA

I

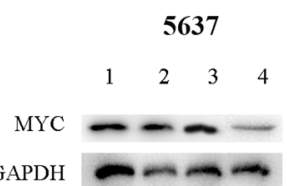

Fig. 4 Effects of synthetic regulatory RNAs on luciferase activity, and MYC mRNA and protein levels. (a, b and c) When aRNA was not expressed in the three cell lines, the luciferase activity was significantly decreased between the NC iRNA group and iRNA group in T24, 5637 and HFF. However, the luciferase activity was still significantly decreased in HFF (a) after addition of aRNA in cells between these two groups. But the luciferase activity was no statistical difference in T24 (b) and 5637 (c). (d, e, f, g, h and i) Without aRNA, there was no difference between column 1 and 2 in all three cell lines and therefore the transcriptional and translational levels of MYC was not inhibited. After the cells were transfected with aRNA as shown in T24 and 5637, the mRNA and protein levels of MYC was largely reduced by expression of MYC amiRNA (column 4 in E, F, $\mathrm{H}$ and I) compared with the negative group (column 3 in $\mathbf{e}, \mathbf{f}, \mathbf{h}$ and $\mathbf{i}$ ). However, there was no significant difference between column 3 and 4 (d and $\mathbf{g})$ in HFF. All data are presented as the mean $\pm \mathrm{SD}\left({ }^{*} p<0.05,{ }^{* *} p<0.01\right)$; bar, SD 
in HFF (Fig. 4A, $\mathrm{P}<0.01$ ). These data suggested that aRNA can bind iRNA to expose UAS again and reverse the repressive effects of iRNA on luciferase activity in T24 and 5637, but not HFF.

\section{The mRNA levels and protein levels of MYC were} selectively inhibited by synthetic regulatory RNAs in bladder cancer cells

As shown in subsequent validated experiments (Fig. 4D, E and $\mathrm{F}$ ), in the absence of aRNA, amiRNAs (NC amiRNA and $M Y C$ amiRNA) were not expressed in all three cell lines and therefore could not inhibit the transcriptional level and protein level of MYC (column 1 and 2 in Fig. 4D, E, F, G, H and I). After cells were transfected with aRNA driven by the mutant $h T E R T$ promoter, the aRNA was only expressed in T24 and 5637, thus interacting with iRNA to expose UAS to allow GAL4-VP64 to combine with UAS. Expectedly, as shown in T24 and 5637, the
mRNA level of $M Y C$ was largely reduced by expressed $M Y C$ amiRNA (column 4 in Fig. 4E, F) compared with the negative group (column 3 in Fig. 4E and F). However, there was no significant difference between column 3 and 4 in Fig. 4D $(P>0.05)$. Besides, similar results were shown in the protein levels of MYC. There was no obvious difference between column 3 and 4 in HFF (Fig. 4G). However, the protein levels of MYC were significantly inhibited in column 4 group compare with column 3 in T24 (Fig. 4H) and 5637 (Fig. 4I). These results demonstrated that this synthetic system can selectively control the expression of the $M Y C$ oncogene in bladder cancer T24 and 5637 cell lines without affecting HFF.

Selective inhibition of cell growth by synthetic regulatory RNAs in bladder cancer cells

A CCK-8 assay was performed to detect cell proliferation. T24, 5637, and HFF were transfected with vectors

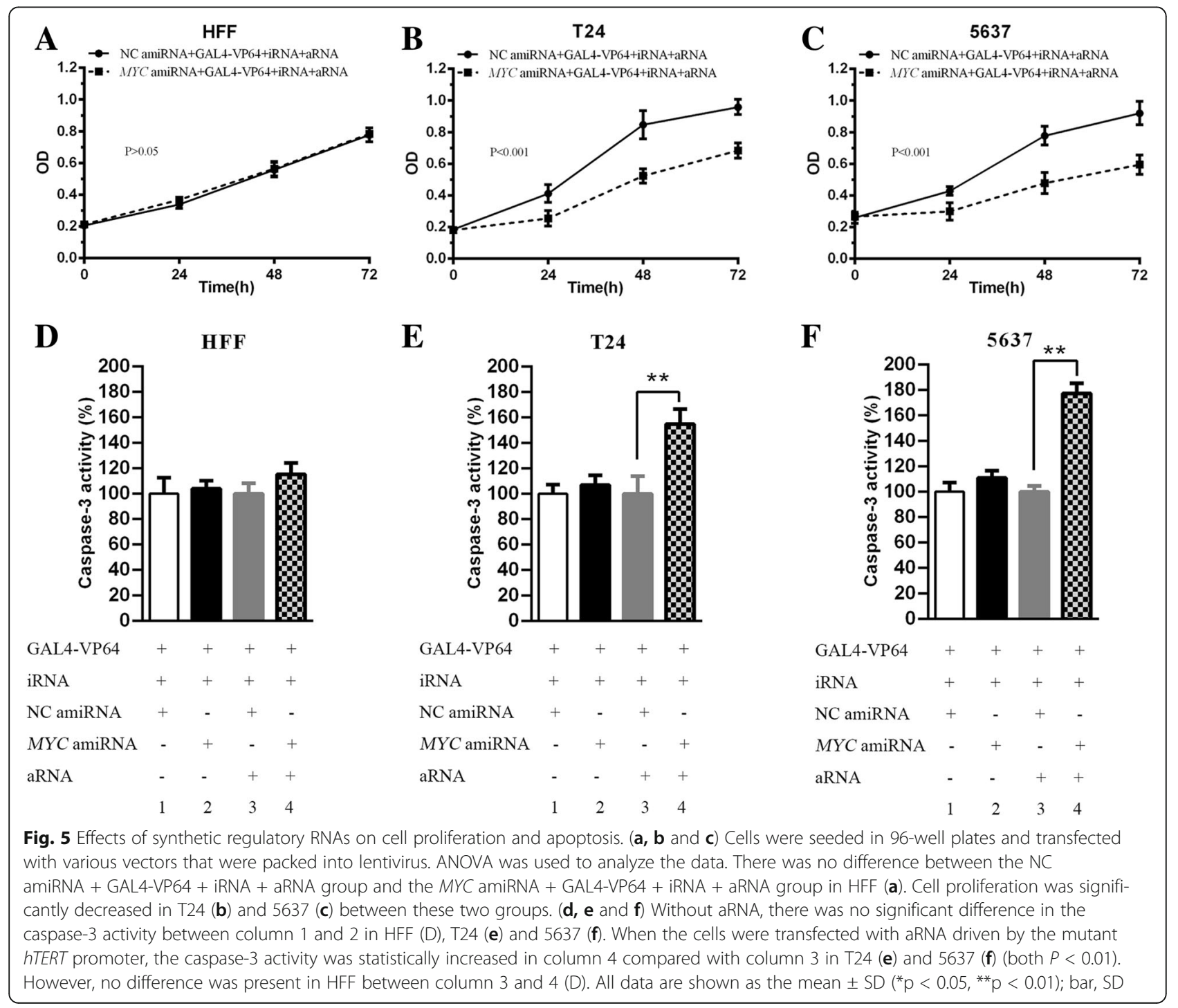


that were packed into lentivirus in 96-well plates. The CCK- 8 assay showed that there was no significant difference between the NC amiRNA + GAL4$\mathrm{VP} 64+$ iRNA + aRNA group and the $M Y C$ amiRNA + GAL4-VP64 + iRNA + aRNA group in HFF (Fig. 5A, P > 0.05). However, cell proliferation was suppressed significantly between these two groups in T24 and 5637 (Fig. 5B and $\mathrm{C}, \mathrm{P}<0.001$ ). The results indicated that cell growth was selectively inhibited by synthetic regulatory RNAs in bladder cancer cells.

\section{Selectively inducing apoptosis by synthetic regulatory RNAs in bladder cancer cells}

To investigate the effect of artificial miRNA and regulatory RNA on the apoptosis of bladder cancer cells and HFF cells, an ELISA assay was performed to measure the apoptosis rate. Without the expression of aRNA, the caspase-3 activity was not different between group 1 (column 1) and group 2 (column 2) in HFF, T24 and 5637 (Fig. 5D, E and F, P > 0.05). When aRNA driven by a mutant $h T E R T$ promoter was added, the caspase-3 activity did not significantly change between group 3 (column 3) and group 4 (column 4) in HFF. However, the caspase- 3 activity increased statistically between group 3 and 4 in T24 and 5637 (Fig. 5E and F, P $<0.01$ ). The data showed that cell apoptosis was selectively promoted by synthetic regulatory RNAs in bladder cancer cells.

\section{Selective inhibition of cell migration by synthetic regulatory RNAs in bladder cancer cells}

A cell scratch assay was used to measure cell motility in 6-well plates according to the manufacturer's protocol. Without the expression of aRNA, compared with the NC amiRNA + GAL4-VP64 + iRNA group, the relative

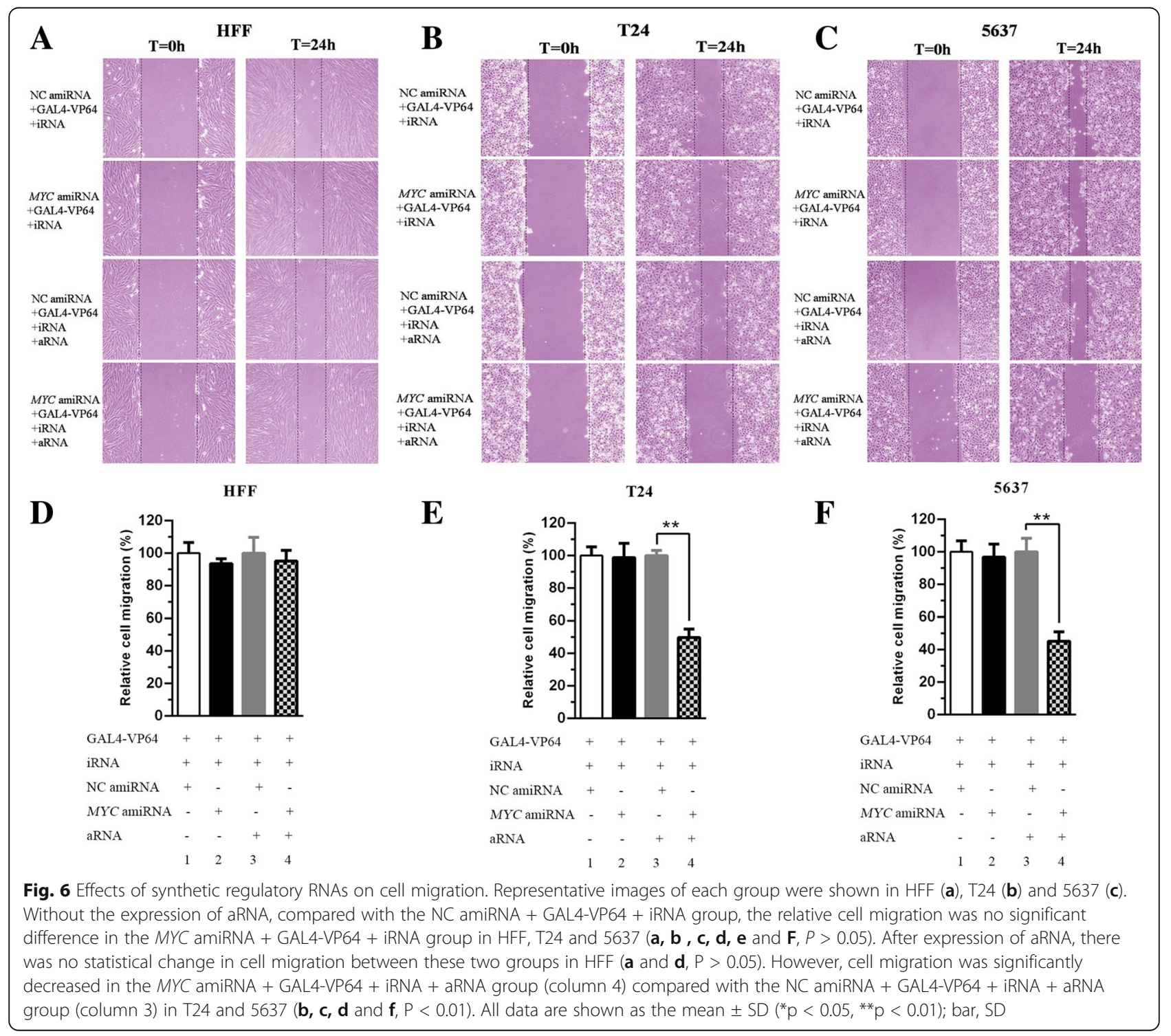


cell migration was not significantly different in the $M Y C$ amiRNA + GAL4-VP64 + iRNA group in HFF, T24 and 5637 (Fig. 6A, B, C, D, E and F, P > 0.05). When aRNA was added to this system, there was no statistical change between the NC amiRNA + GAL4-VP64 + iRNA + aRNA group and the $M Y C$ amiRNA + GAL4-VP64 + iRNA + aRNA group in HFF (Fig. 6A and D, P > 0.05). However, cell migration was significantly decreased in the $M Y C$ amiRNA + GAL4-VP64 + iRNA + aRNA group (column 4) compared with the NC amiRNA + GAL4VP64 + iRNA + aRNA group (column 3) in T24 and 5637 (Fig. 6B, C, E and F, P < 0.01). The cell migration was inhibited by approximately $50 \%$ in T24 (Fig. 6E, P < 0.01 ) and approximately $55 \%$ in 5637 (Fig. 6F, $\mathrm{P}<$ 0.01 ). The cell migration results revealed that cell mobility was selectively suppressed by synthetic regulatory RNAs in bladder cancer cells.

\section{Knockdown of MYC by synthetic regulatory RNAs inhibited bladder cancer cell growth in vivo}

To examine the knockdown effect of MYC by synthetic regulatory RNAs, vectors were packed into lentvirus. To measure the functional role of synthetic regulatory RNAs in tumor growth in vivo, 5637 cells expressing LV-Treatment or LV-NC were subcutaneously inoculated into nude mice. Thirty days later, tumors were harvested and the tumor volume was significantly smaller than that in LV-Treatment compared with the LV-NC group (Fig. 7A). As shown in Fig. 7B, the tumor volume curve was significantly suppressed in LV-Treatment group. Besides, the tumor weight in LV-Treatment group was obviously lower than that in LV-NC group (Fig. 7C, $\mathrm{P}<0.01$ ). In summary, knockdown of MYC by synthetic regulatory RNAs can inhibit bladder cancer cell growth in vivo. These results were consistent with the findings of cell growth assay by CCK- 8 .

\section{Discussion}

RNA molecules are often regarded as messengers of information from genes to the protein and also have regulatory roles in human diseases [34-37]. Repressor RNA and activator RNA are small RNAs that have regulatory roles in controlling post-transcriptional gene expression in prokaryotic cells $[10,38]$. Whether these regulatory RNAs can control gene expression in eukaryotic cells is unknown. In our study, we construct regulatory RNAs, iRNA and aRNA according to the principles of creation in a previous study [10], and it is the first time to measure the roles of regulatory RNAs in mammal cells.

UAS cannot drive the downstream gene expression without the binding of activating proteins, such as GAL4 or GAL4-VP64 fusion protein [13, 39]. In the present study, the iRNA was used to compete with GAL4-VP64 protein to bind UAS. However, synthetic aRNA interacted with iRNA and exposed UAS again. Thus, GAL4VP64 protein can bind UAS again and the downstream gene of UAS could be expressed.

The amiRNAs were inserted into the downstream of UAS in this project. MicroRNAs (miRNAs) are small
A

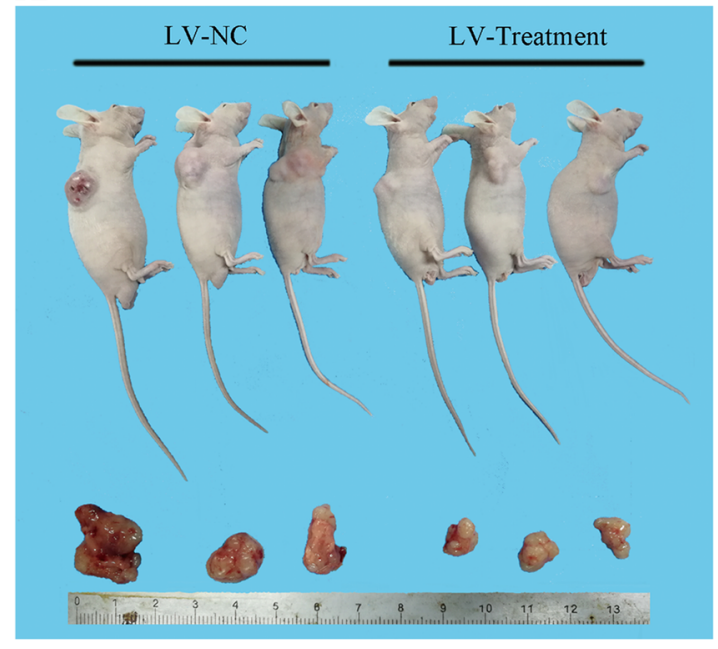

B

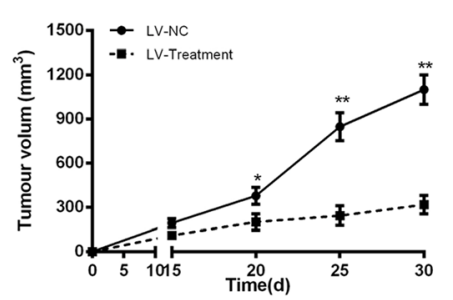

$\mathbf{C}$

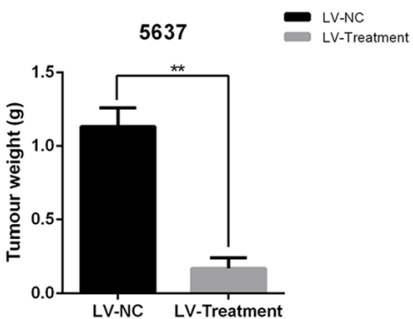

Fig. 7 Effects of synthetic regulatory RNAs on cell growth in vivo. (a) Representative images of tumor were shown in LV-NC group and LVTreatment group. LV-NC group represents NC amiRNA + GAL4-VP64 + iRNA + aRNA. LV-Treatment group is on behalf of MYC amiRNA + GAL4VP64+ iRNA + aRNA. (b) Compared with the LV-NC group, the tumor volume curve was significantly suppressed in LV-Treatment group. (c) Compared with the LV-NC group, the tumor weight in LV-Treatment group was obviously lower than that in LV-NC group $(P<0.01)$. All data are shown as the mean \pm SD $\left({ }^{*} p<0.05,{ }^{*} p<0.01\right)$; bar, SD 
non-coding RNAs and play significant roles in many biological processes [5, 40-42]. Studies show that artificial miRNAs can promote gene silencing in a similar manner to natural miRNAs and they have the same efficiency and are more stable and less cytotoxic compared with shRNAs or siRNAs [43, 44]. Artificial miRNAs can be designed and synthesized to silence multiple genes or a cluster of amiRNA sequences can be constructed to efficiently suppress one gene [9]. Owing to the vital oncogenic role of MYC in the metabolism of cancer, we choose MYC as the therapeutic target for future study.

The mutant $h T E R T$ promoter is a potential tumorspecific element and has been used to selectively drive expression of downstream genes in bladder cancer cells [17]. Therefore, trying to solve limitations in the specificity and effectiveness of treatment in bladder cancer, we also take advantage of this element to selectively control gene expression in bladder cancer cells. In this study, the aRNA was driven by the mutant hTERT promoter picked from a previous study [19] and expressed in bladder cancer cells but not human foreskin fibroblast cells. In our work, expression of the amiRNA targeting MYC was only activated when GAL4-VP64 fusion protein binds UAS. We used synthetic regulatory RNAs to control this binding procedure (GAL4-VP64 binds UAS). Compared with their work [19], our strategy has two advantages. The p65 or VPR (a chimeric activator that is composed of the VP64, p65 and Rta domains) showed much higher transcriptional activation efficiency than VP64 [45]. Our strategy is modular and we could replace GAL4-VP64 with GAL4-P65 or GAL4-VPR to get much higher driven efficiency for expression of the downstream gene in the future, which is one merit. What's more, stable expression of amiRNAs targeting MYC may be harmful for cells and we construct synthetic regulatory RNAs that are regarded as a similarly "switch" to control the expression of amiRNA, which is the other advantage.

We verified the function of $M Y C$ in bladder cancer cells. High expression of $M Y C$ was significantly correlated with bladder cancer histological grade and TNM stage. Additionally, functional experiments showed that $M Y C$ is an oncogene in bladder cancer cells. Then, we constructed tandem amiRNA sequences targeting oncogenic $M Y C$ in bladder cancer driven by UAS and tested whether synthetic regulatory RNAs can regulate the expression of amiRNA. Our results demonstrated that when cells express iRNA and GAL4-VP64 protein in bladder cancer cells and HFF cells, the expression of $M Y C$ cannot be significantly inhibited. When aRNA driven by a tumor-specific promoter was transiently transfected into cells, amiRNA targeting $M Y C$ can be expressed to markedly decrease the mRNA and protein expression levels of oncogenic $M Y C$ and significantly inhibit cell growth in vitro, induce apoptosis and suppress the migration of bladder cancer cells, but not human foreskin fibroblast cells. What's more, the in vivo experiment showed that expression of aRNA can inhibit tumor volume compared with the relative negative control.

In conclusion, we used a synthetic platform to design and construct synthetic regulatory RNAs and artificial miRNAs. We can selectively control the expression of synthetic artificial miRNAs to inhibit progression of bladder cancer by regulatory iRNA and aRNA in vitro and in vivo. Synthetic regulatory RNAs might be a selective therapeutic method for bladder cancer.

\section{Acknowledgements}

We thank all of the donors whose names were not included in the author list, but who participated in this program. This work was supported by grants from the Shenzhen Project of Science and Technology

(JCYJ20170307111748761 and 201506042).

Competing interest

The authors declare that they have no competing interest.

\section{Publisher's Note}

Springer Nature remains neutral with regard to jurisdictional claims in published maps and institutional affiliations.

\section{Author details}

${ }^{1}$ Guangdong and Shenzhen Key Laboratory of Male Reproductive Medicine and Genetics, Institute of Urology, Peking University Shenzhen Hospital, Shenzhen-Peking University-the Hong Kong University of Science and Technology Medical Center, Shenzhen 518000, People's Republic of China. ${ }^{2}$ The Department of Biochemistry and Molecular Biology, School of Basic Medical Sciences, Peking University Health Science Center, Beijing 100191, People's Republic of China.

Received: 27 June 2017 Accepted: 23 October 2017

Published online: 30 October 2017

\section{References}

1. Jacobs BL, Lee CT, Montie JE. Bladder cancer in 2010: how far have we come? CA Cancer J Clin. 2010;60:244-72.

2. Marta GN, Hanna SA, Gadia R, Correa SF, Silva JL, Carvalho Hde A. The role of radiotherapy in urinary bladder cancer: current status. Int Braz J Urol. 2012;38:144-53. discussion 153-144

3. Racioppi M, D'Agostino D, Totaro A, Pinto F, Sacco E, D'Addessi A, et al. Value of current chemotherapy and surgery in advanced and metastatic bladder cancer. Urol Int. 2012;88:249-58.

4. Bartel DP. MicroRNAs: target recognition and regulatory functions. Cell. 2009;136:215-33.

5. Sayed D, Abdellatif M. MicroRNAs in development and disease. Physiol Rev. 2011;91:827-87.

6. Luo H, Yang R, Li C, Tong Y, Fan L, Liu X, et al. MicroRNA-139-5p inhibits bladder cancer proliferation and self-renewal by targeting the Bmi1 oncogene. Tumour Biol. 2017;39:1010428317718414.

7. Krol J, Loedige I, Filipowicz W. The widespread regulation of microRNA biogenesis, function and decay. Nat Rev Genet. 2010;11:597-610.

8. Tang $L$, Chen HY, Hao LB, Tang B, Guo H, Yong $X$, et al. microRNA inhibitors: natural and artificial sequestration of microRNA. Cancer Lett. 2017;

9. Wang T, Xie Y, Tan A, Li S, Xie Z. Construction and characterization of a synthetic MicroRNA cluster for multiplex RNA interference in mammalian cells. ACS Synth Biol. 2016;5:1193-200.

10. Isaacs FJ, Dwyer DJ, Ding C, Pervouchine DD, Cantor CR, Collins JJ. Engineered riboregulators enable post-transcriptional control of gene expression. Nat Biotechnol. 2004;22:841-7. 
11. Brand AH, Perrimon N. Targeted gene expression as a means of altering cell fates and generating dominant phenotypes. Development. 1993;118:401-15.

12. Asakawa K, Kawakami K. Targeted gene expression by the Gal4-UAS system in zebrafish. Develop Growth Differ. 2008;50:391-9.

13. Chen X, Li T, Wang X, Du Z, Liu R, Yang Y. Synthetic dual-input mammalian genetic circuits enable tunable and stringent transcription control by chemical and light. Nucleic Acids Res. 2016;44:2677-90.

14. Harley CB. Telomerase and cancer therapeutics. Nat Rev Cancer. 2008;8:167-79.

15. Verdun RE, Karlseder J. Replication and protection of telomeres. Nature. 2007:447:924-31.

16. Wu S, Huang P, Li C, Huang Y, Li X, Wang Y, et al. Telomerase reverse transcriptase gene promoter mutations help discern the origin of urogenital tumors: a genomic and molecular study. Eur Urol. 2014;65:274-7.

17. Zhuang CL, Fu X, Liu L, Liu YC, Huang WR, Cai ZM. Synthetic miRNA sponges driven by mutant hTERT promoter selectively inhibit the progression of bladder cancer. Tumour Biol. 2015;36:5157-63.

18. Sahm F, Schrimpf D, Olar A, Koelsche C, Reuss D, Bissel J, et al. TERT promoter mutations and risk of recurrence in meningioma. J Natl Cancer Inst. 2016:108.

19. Liu L, Liu Y, Zhang T, Wu H, Lin M, Wang C, et al. Synthetic Bax-anti Bcl2 combination module actuated by super artificial hTERT promoter selectively inhibits malignant phenotypes of bladder cancer. J Exp Clin Cancer Res. 2016:35:3.

20. Xie Z, Wroblewska L, Prochazka L, Weiss R, Benenson Y. Multi-input RNAi-based logic circuit for identification of specific cancer cells. Science. 2011;333:1307-11.

21. Liu Y, Zeng Y, Liu L, Zhuang C, Fu X, Huang W, et al. Synthesizing AND gate genetic circuits based on CRISPR-Cas9 for identification of bladder cancer cells. Nat Commun. 2014;5:5393.

22. Chen M, Zhuang C, Liu Y, Li J, Dai F, Xia M, et al. Tetracycline-inducible shRNA targeting antisense long non-coding RNA HIF1A-AS2 represses the malignant phenotypes of bladder cancer. Cancer Lett. 2016;376:155-64.

23. Risuleo G, Ciacciarelli M, Castelli M, Galati G. The synthetic inhibitor of fibroblast growth factor receptor PD166866 controls negatively the growth of tumor cells in culture. J Exp Clin Cancer Res. 2009;28:151.

24. Kashyap N, Pham B, Xie Z, Bleris L. Transcripts for combined synthetic microRNA and gene delivery. Mol BioSyst. 2013;9:1919-25.

25. Premsrirut PK, Dow LE, Kim SY, Camiolo M, Malone CD, Miething C, et al. A rapid and scalable system for studying gene function in mice using conditional RNA interference. Cell. 2011;145:145-58.

26. McBride JL, Boudreau RL, Harper SQ, Staber PD, Monteys AM, Martins I, et al. Artificial miRNAs mitigate shRNA-mediated toxicity in the brain: implications for the therapeutic development of RNAi. Proc Natl Acad Sci U S A. 2008;105:5868-73.

27. Stegmeier F, Hu G, Rickles RJ, Hannon GJ, Elledge SJ. A lentiviral microRNAbased system for single-copy polymerase II-regulated RNA interference in mammalian cells. Proc Natl Acad Sci U S A. 2005;102:13212-7.

28. Fellmann C, Hoffmann T, Sridhar V, Hopfgartner B, Muhar M, Roth M, et al. An optimized microRNA backbone for effective single-copy RNAi. Cell Rep. 2013:5:1704-13

29. Zack TI, Schumacher SE, Carter SL, Cherniack AD, Saksena G, Tabak B, et al. Pan-Cancer patterns of somatic copy number alteration. Nat Genet. 2013:45:1134-40.

30. Stine ZE, Walton ZE, Altman BJ, Hsieh AL, Dang CV. MYC, metabolism, and cancer. Cancer Discov. 2015:5:1024-39.

31. Watters AD, Latif Z, Forsyth A, Dunn I, Underwood MA, Grigor KM, et al. Genetic aberrations of c-myc and CCND1 in the development of invasive bladder cancer. Br J Cancer. 2002;87:654-8.

32. Schultz L, Albadine R, Hicks J, Jadallah S, DeMarzo AM, Chen YB, et al. Expression status and prognostic significance of mammalian target of rapamycin pathway members in urothelial carcinoma of urinary bladder after cystectomy. Cancer. 2010;116:5517-26.

33. Zhuang C, Li J, Liu Y, Chen M, Yuan J, Fu X, et al. Tetracycline-inducible shRNA targeting long non-coding RNA PVT1 inhibits cell growth and induces apoptosis in bladder cancer cells. Oncotarget. 2015;6:41194-203.

34. Eddy SR. Non-coding RNA genes and the modern RNA world. Nat Rev Genet. 2001;2:919-29.

35. Fendler A, Stephan C, Yousef GM, Kristiansen G, Jung K. The translationa potential of microRNAs as biofluid markers of urological tumours. Nat Rev Urol. 2016:13:734-52

36. Joyce GF. The antiquity of RNA-based evolution. Nature. 2002;418:214-21.
37. Cho WC. MicroRNAs: potential biomarkers for cancer diagnosis, prognosis and targets for therapy. Int J Biochem Cell Biol. 2010;42:1273-81.

38. Krishnamurthy M, Hennelly SP, Dale T, Starkenburg SR, Marti-Arbona R, Fox DT, et al. Tunable Riboregulator switches for post-transcriptional control of gene expression. ACS Synth Biol. 2015;4:1326-34.

39. Jones WD. The expanding reach of the GAL4/UAS system into the behavioral neurobiology of drosophila. BMB Rep. 2009;42:705-12.

40. Xie Y, Ma X, Chen L, Li H, Gu L, Gao Y, et al. MicroRNAs with prognostic significance in bladder cancer: a systematic review and meta-analysis. Sci Rep. 2017;7:5619.

41. Wang J, Zhao X, Shi J, Pan Y, Chen Q, Leng P, et al. miR-451 suppresses bladder cancer cell migration and invasion via directly targeting c-Myc. Oncol Rep. 2016:36:2049-58.

42. Cho WC. MicroRNAs as therapeutic targets and their potential applications in cancer therapy. Expert Opin Ther Targets. 2012;16:747-59.

43. Calloni R, Bonatto D. Scaffolds for artificial miRNA expression in animal cells. Hum Gene Ther Methods. 2015;26:162-74.

44. Ossowski S, Schwab R, Weigel D. Gene silencing in plants using artificial microRNAs and other small RNAs. Plant J. 2008;53:674-90.

45. Chavez A, Scheiman J, Vora S, Pruitt BW, Tuttle M, lyere E PR, et al. highly efficient Cas9-mediated transcriptional programming. Nat Methods. 2015;12:326-8.

\section{Submit your next manuscript to BioMed Central and we will help you at every step:}

- We accept pre-submission inquiries

- Our selector tool helps you to find the most relevant journal

- We provide round the clock customer support

- Convenient online submission

- Thorough peer review

- Inclusion in PubMed and all major indexing services

- Maximum visibility for your research

Submit your manuscript at www.biomedcentral.com/submit
Biomed Central 